\title{
Cephalic Pancreaticoduodenectomy for Bleeding Duodenal Arteriovenous Malformation
}

\author{
Ruben Ortiz $^{1}$ Eva Dominguez ${ }^{1} \quad$ S. Barrena ${ }^{1}$ Leopoldo Martinez ${ }^{1}$ Gerardo Prieto ${ }^{2}$ Emilio Burgos ${ }^{3}$ \\ Juan Antonio Tovar ${ }^{1}$ \\ ${ }^{1}$ Department of Pediatric Surgery, Hospital Universitario La Paz, \\ Idipaz, Madrid, Spain \\ 2 Department of Pediatric Gastroenterology, Hospital Universitario La \\ Paz, Madrid, Spain \\ ${ }^{3}$ Department of Pathology, Hospital Universitario La Paz, \\ Madrid, Spain \\ Eur J Pediatr Surg Rep 2014;2:13-15. \\ Address for correspondence Ruben Ortiz, MD, Department of \\ Pediatric Surgery, Hospital Universitario La Paz, Idipaz, Paseo de la \\ Castellana 261, Madrid 28046, Spain \\ (e-mail: rubenortizrodriguez@hotmail.com).
}

\author{
Abstract \\ Keywords \\ - gastrointestinal \\ bleeding \\ - vascular \\ malformation \\ - pancreaticod- \\ uodenectomy \\ - arteriovenous \\ malformation
}

Introduction Treatment of recurrent severe gastrointestinal bleeding due to arteriovenous malformations may require complex resections. In some particular locations, extensive surgery is the only way out, as shown in this report.

Case Report A 2.5-year-old child suffered repeated episodes of upper gastrointestinal bleeding since the first month of life. After an extensive diagnostic workout, the diagnosis of duodenal arteriovenous malformation was established. Cephalic pancreaticoduodenectomy with pyloric preservation was performed and no further episodes of bleeding occurred in the ensuing 2 years.

Conclusion Bleeding malformations located in the pancreaticoduodenal area can be effectively treated in children by pylorus-preserving cephalic pancreaticoduodenectomy.

\section{Introduction}

Intestinal vascular malformations are rare in childhood. ${ }^{1}$ They can affect any intestinal segment and may remain asymptomatic. They can be diagnosed upon workup for gastrointestinal bleeding, recurrent abdominal pain, intestinal obstruction, or intussusception. ${ }^{1,2}$ These lesions are rarely accompanied by other vascular malformations. ${ }^{1-3}$

Treatment may include endoscopic fulguration, surgical excision, or bowel resection. Sometimes, this resection is only possible by sacrificing adjacent structures. The purpose of this report is to demonstrate that this radical approach is justified when the malformation is located in the duodenopancreatic area, where more conservative procedures are not feasible.

\section{Case Report}

A 2.5-year-old child, with no history of previous disease, started at 12 months of age with repeated episodes of upper gastrointestinal bleeding requiring frequent transfusions.
Several upper fiberoptic endoscopies found no bleeding points, but showed an apparently inflammatory redness of the duodenal mucosa. Considering the presence of atopic dermatitis and positive skin prick tests to egg and cow's milk, food allergy was suspected; however, a restricted diet brought no relief and bleeding continued.

A 99mTechnetium (99mTc) scintigraphy showed no pathological uptake. Capsule endoscopy depicted a pattern of duodenitis, and $99 \mathrm{mTc}$-labeled red cell scintigraphy showed a fleeting image in the left abdomen. Contrast computed tomographic (CT) scan demonstrated a $2-\mathrm{cm}$ tumor in the wall of the second portion of the duodenum; intravenous contrast showed markedly increased vascularization in this region ( - Fig. 1a). Selective pancreaticoduodenal arteriography showed a blush in the papillary region with rapid venous filling consistent with the diagnosis of arteriovenous malformation (- Fig. 1b).

At laparotomy, full thickness wall biopsies showed dysplastic vessels in the mucosa and submucosa (-Fig. 2a). Removal of the entire duodenum along with the head of the received

July 17, 2013

accepted after revision

August 16, 2013

published online

January 16, 2014
DOI http://dx.doi.org/

10.1055/s-0033-1357263. ISSN 2194-7619. (c) 2014 Georg Thieme Verlag KG Stuttgart · New York
License terms

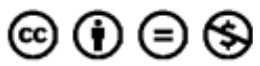



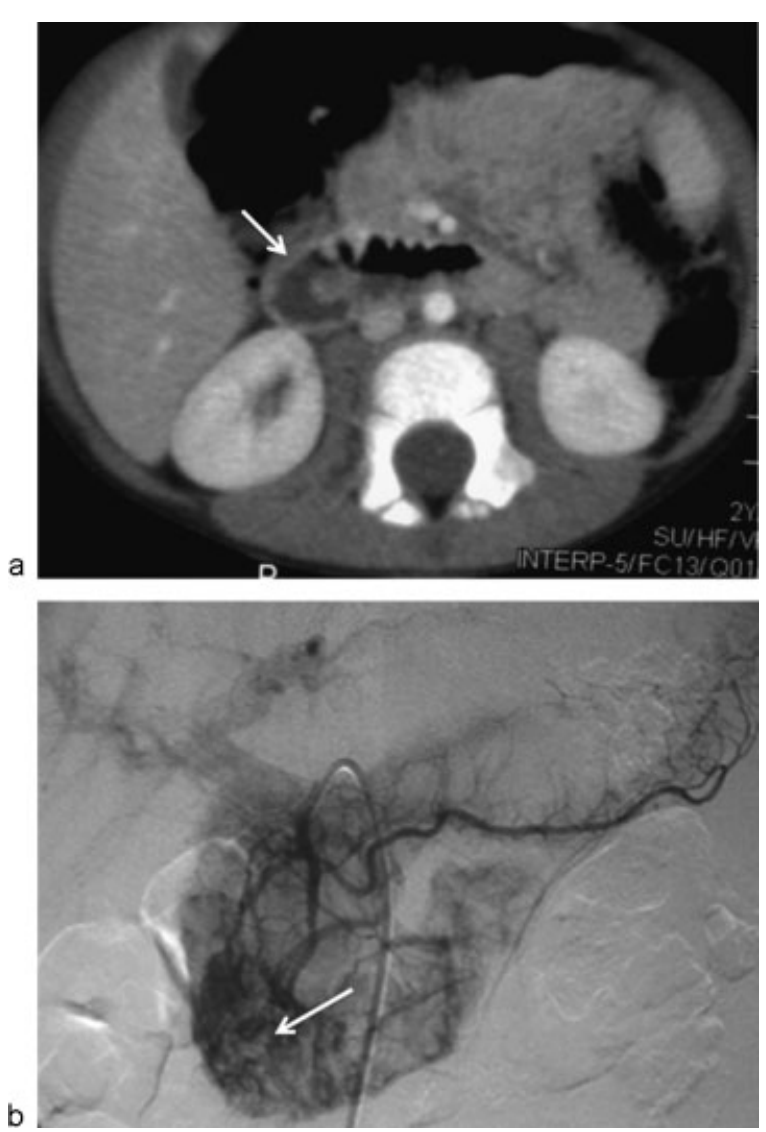

Fig. 1 (a) An abdominal computed tomographic scan showing a tumor in the medial wall of the second portion of the duodenum and the neighboring part of the pancreas. Intravenous contrast injection showed markedly increased vascularity in this region. (b) Selective angiography: Increased blood flow at the level of the gastroduodenal artery and first jejunal branches of the superior mesenteric artery is observed, with early venous outflow to the portal vein, consistent with the diagnosis of arteriovenous malformation.

pancreas was then decided. A pyloric-preserving cephalic pancreaticoduodenectomy with biliary and pancreatic diversion into a Roux-en-Y jejunal loop was performed (-Fig. 3).

Pathological study showed a thickened duodenal submucosa in the papilla and surrounding areas, with multiple arterial and venous vessels. The pancreas and biliary tract were normal. These findings confirmed the diagnosis of arteriovenous malformation of the duodenum ( - Fig. 2b).

Three years after the procedure, the patient lives a normal life except for the exclusion diet due to food allergy. Anemia and gastrointestinal bleeding disappeared after surgery and never recurred.

\section{Discussion}

Gastrointestinal bleeding, secondary to intestinal vascular malformations, occurs rarely in children. ${ }^{1}$ Their description in the literature is confusing making appropriate classification and prognosis difficult. ${ }^{1,3}$ For diagnostic purposes, ultrasound, upper gastrointestinal endoscopy, and barium enema
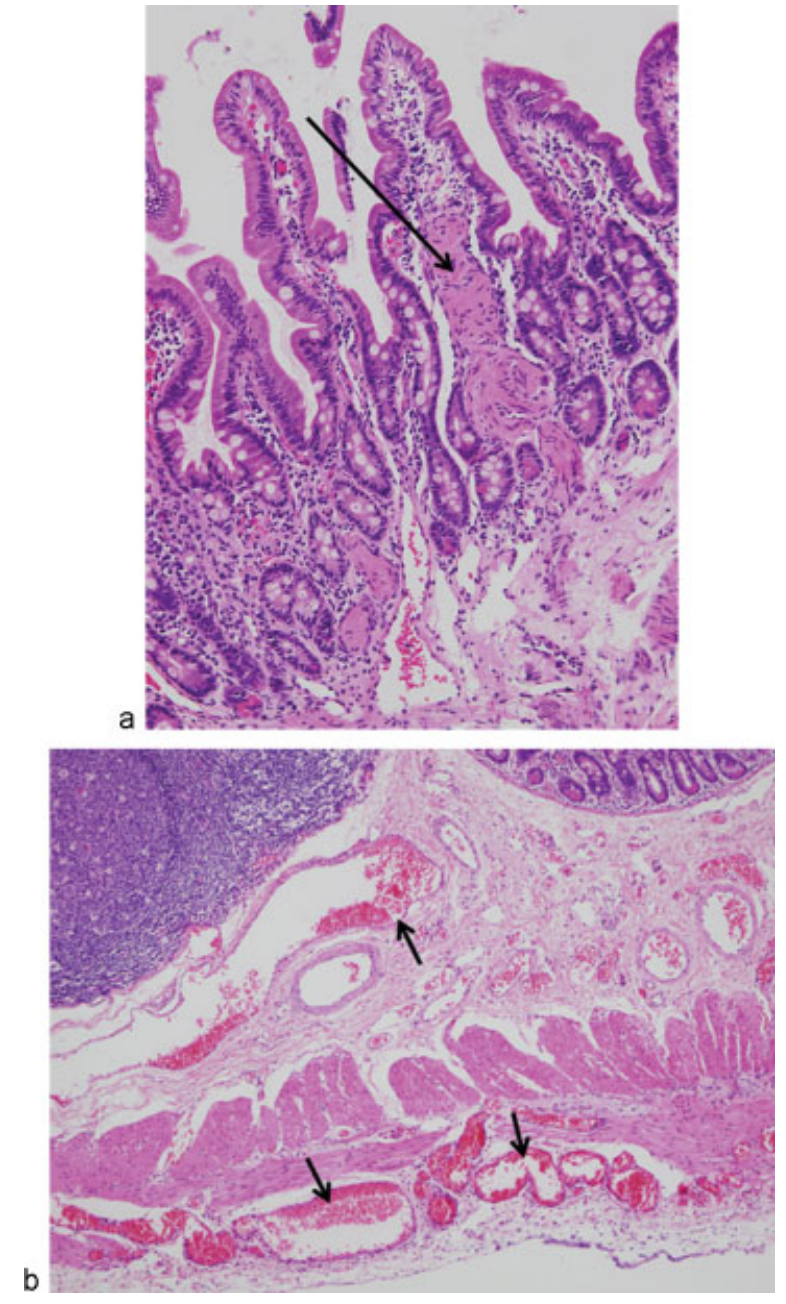

Fig. 2 (a) Endoscopic duodenojejunal biopsy: At the level of the submucosa, a conglomerate of thick-walled hamartomatous vessels is clearly observed. (b) Operative specimen of the ampullary region: Several tortuous vessels, with arterial and venous patterns, suggestive of arteriovenous malformation are seen in the submucosa.

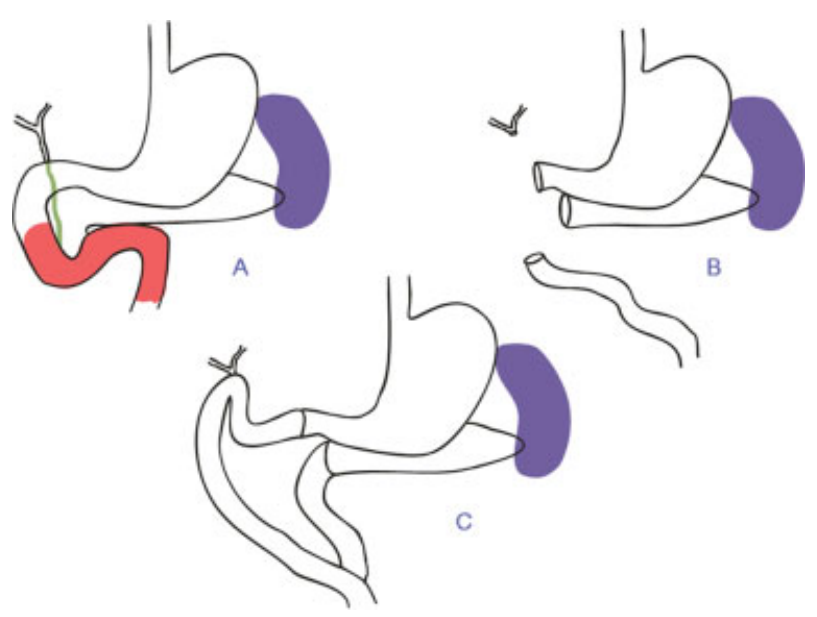

Fig. 3 Schematic diagram of pancreaticoduodenectomy: (A and B) The entire duodenum was removed along with the head of the pancreas; (C) a pylorus-preserving pancreaticoduodenectomy, with biliary and pancreatic diversion into a Roux-en-Y jejunal loop was performed. 
are rarely useful. 99mTc scintigraphy is especially helpful in active bleeding. ${ }^{1,3-5}$ A CT scan with intravenous contrast, capsule endoscopy and intraoperative enteroscopy can also facilitate the diagnosis, and selective arteriography may define their location and extent. This should be done in patients with suspected intestinal vascular malformation, when other tests are inconclusive; otherwise, they can be missed during surgery. $1,3,6$

When bleeding cannot be controlled by endoscopic procedures, a simultaneous selective arterial embolization can be useful. $^{1,2,4,6}$ In the present case, the nature and localization of the malformation led to the performance of a Whipple procedure. This operation is rarely used in children except for removal of pancreatic tumors, ${ }^{6-9}$ and it is considered a risky procedure in adults with a mortality ranging between 2 and $13 \%{ }^{9}$

We are not aware of any other cases of isolated duodenal vascular malformations treated in this manner. However, this modality of treatment has been used for the treatment of vascular anomalies located in the pancreas and adjacent tissues. $^{5}$

In our opinion, such radical procedure may be justified in cases of severe bleeding due to duodenal vascular malformations.

\section{References}

1 Aziz A, Kane TD, Meza MP, Vaughan KG, Hackam DJ. An unusual cause of rectal bleeding and intestinal obstruction in a child with peripheral vascular malformations. Pediatr Surg Int 2005;21(6):491-493

2 Gordon FH, Watkinson A, Hodgson H. Vascular malformations of the gastrointestinal tract. Best Pract Res Clin Gastroenterol 2001; 15(1):41-58

3 Frémond B, Yazbeck S, Dubois J, Brochu P, Garel L, Ouimet A. Intestinal vascular anomalies in children. J Pediatr Surg 1997; 32(6):873-877

4 Sugito K, Kusafuka T, Hoshino M, et al. Usefulness of color Doppler sonography and $99 \mathrm{~m}$ Tc-RBC scintigraphy for preoperative diagnosis of a venous malformation of the small intestine in a 2-yearold child. J Clin Ultrasound 2008;36(1):56-58

5 Vogel AM, Alesbury JM, Fox VL, Fishman SJ. Complex pancreatic vascular anomalies in children. J Pediatr Surg 2006;41(3):473-478

6 Park J, Dunn JC, Atkinson JB. Management of children with pancreatic head mass. J Pediatr Surg 2006;41(6):e1-e4

7 Newman K, Vates T, Duffy L, Anderson K. Pancreatoduodenectomy with preservation of the stomach and pylorus: a safe and effective alternative in children. J Pediatr Surg 1992;27(10):1334-1335

8 Ohata R, Okazaki T, Ishizaki Y, et al. Pancreaticoduodenectomy for pancreatoblastoma: a case report and literature review. Pediatr Surg Int 2010;26(4):447-450

9 Uchida K, Joseph JM, Gapany C, Chardot C. Modified digestive reconstruction with midgut transposition after pylorus-preserving pancreaticoduodenectomy for pancreatic head tumor in childhood. J Pediatr Surg 2008;43(10):1932-1934 